\title{
Winds from stripped low-mass helium stars and Wolf-Rayet stars
}

\author{
Jorick S. Vink
}

\author{
Armagh Observatory and Planetarium, College Hill, Armagh, BT61 9DG, UK \\ e-mail: jsv@arm.ac.uk
}

Received 6 September 2017 / Accepted 12 October 2017

\begin{abstract}
We present mass-loss predictions from Monte Carlo radiative transfer models for helium $(\mathrm{He})$ stars as a function of stellar mass, down to $2 M_{\odot}$. Our study includes both massive Wolf-Rayet (WR) stars and low-mass He stars that have lost their envelope through interaction with a companion. For these low-mass He stars we predict mass-loss rates that are an order of magnitude smaller than by extrapolation of empirical WR mass-loss rates. Our lower mass-loss rates make it harder for these elusive stripped stars to be discovered via line emission, and we should attempt to find these stars through alternative methods instead. Moreover, lower massloss rates make it less likely that low-mass He stars provide stripped-envelope supernovae (SNe) of type Ibc. We express our mass-loss predictions as a function of $L$ and $Z$ and not as a function of the He abundance, as we do not consider this physically astute given our earlier work. The exponent of the $\dot{M}$ versus $Z$ dependence is found to be 0.61 , which is less steep than relationships derived from recent empirical atmospheric modelling. Our shallower exponent will make it more challenging to produce "heavy" black holes of order $40 M_{\odot}$, as recently discovered in the gravitational wave event GW 150914, making low metallicity for these types of events even more necessary.
\end{abstract}

Key words. stars: early-type - stars: black holes - stars: massive - stars: mass-loss - stars: winds, outflows - stars: Wolf-Rayet

\section{Introduction}

We present mass-loss predictions for helium (He) stars, including both massive Wolf-Rayet (WR) stars, and low-mass He stars that have presumably lost their hydrogen $(\mathrm{H})$ envelope through binary mass transfer (e.g. Podsiadlowski et al. 1992).

One of the main ingredients for massive star evolution modelling concerns their rates of stellar wind mass loss (Langer 2012; Yoon et al. 2012; Georgy et al. 2013; Limongi \& Chieffi 2006). For the H-burning main sequence, we are in the fortunate position that a theoretical recipe such as that by Vink et al. $(2000,2001)$ can be utilized in a meaningful way, but for more evolved stars, such as WR stars, the community still needs to rely on empirical recipes, such as the recipe by Nugis \& lamers (2000). A notable drawback of employing empirical recipes is that they cannot be applied in a parameter space for which they have not been derived. These recipes might fail dramatically outside that parameter space, for example for low-mass He-stripped stars, especially when the recipe has not been constructed from the physically correct stellar parameters (Vink \& de Koter 2002; Puls et al. 2008). One of the main aims of this study is to alleviate this shortcoming.

Reliable mass-loss rates are fundamental to our understanding of the late evolution of massive stars towards core collapse, either as supernovae (SNe) Ibc, or direct black hole formation (Eldridge \& Vink 2006; Belczynski et al. 2010). Such mass-loss rates are especially relevant for understanding merging black holes in recent gravitational wave events such as GW 150914 (Abbott et al. 2016). These rates are also fundamental for correctly predicting the ionizing radiation of these hot stars with major consequences for interpreting He II line emission at intermediate and high red shifts as Population III stars (Cassata et al.2013; Sobral et al. 2015) or very massive stars
(VMS) at low metallicity (Gräfener \& Vink 2015; Szécsi et al. 2015).

There has been much recent attention to detecting the elusive binary progenitors of Ibc supernovae. These objects should be stripped stars that in some sense resemble classical WR stars, but because of their weaker winds may either have very little line emission (Götberg et al. 2017) or no line emission at all. Addressing this question is one of the main aims of this paper.

Mass loss from massive stars is driven by radiative forces on spectral lines (Lucy \& Solomon 1970; Castor et al. 1975; CAK). The CAK work developed the force multiplier formalism to treat all relevant ionic transitions. This enabled these authors to predict the wind mass-loss rate, $\dot{M}$, and terminal velocity, $v_{\infty}$, simultaneously. Using a "global energy" Monte Carlo approach (Abbott \& Lucy 1985) in which the velocity law was adopted - aided by empirical constraints - Vink et al. (2000) predicted mass-loss rates for galactic O-type stars that include multi-line scattering. Müller \& Vink (2008) subsequently introduced a new parametrization of the line acceleration, expressing it as a function of radius instead of the velocity gradient (as in the CAK theory). The implementation of this new formalism improved the local consistency of Monte Carlo models that initially assumed a velocity law. Not only do we find reasonably good agreement with observation of wind terminal velocities, but because our method naturally accounts for the physics of multi-line scattering, it is also applicable to denser winds, such as those of WR stars (Vink et al. 2011a; Vink 2015).

This paper is organized as follows. In Sect. 2, we briefly describe the Monte Carlo modelling and parameter space considered for this study. The mass-loss predictions (Sect. 4) are followed by a discussion, before ending with a summary in Sect. 7 . 


\section{Monte Carlo modelling}

Helium star mass-loss rates are calculated with a Monte Carlo method, in which we follow the fate of a large number of photon packets $\left(2 \times 10^{6}\right)$ from below the stellar photosphere throughout the entire wind, up to the terminal velocity. The core of the method is linked to the total loss of radiative energy that is coupled to the momentum gain of the outflowing gas (Abbott \& Lucy 1985; Vink et al. 1999). As the photon absorptions and scatterings depend on wind density and hence on the mass-loss rate $\dot{M}$, we are able to obtain a consistent model where the momentum of the gas equals the transferred momentum by photons. Here we shall employ our local dynamical approach (Müller \& Vink 2008, 2014; Muijres et al. 2012), in which we predict $\dot{M}$, $v_{\infty}$, and the wind structure parameter, $\beta$, simultaneously.

The Monte Carlo code employs the density and temperature stratifications from a model atmosphere calculation performed with ISA-WIND (de Koter et al. 1993). These model atmospheres are unified, implying continuity between the photosphere and wind region. The models describe the radiative transfer in spectral lines adopting an improved Sobolev approximation that includes the diffuse radiation field. The species that are explicitly calculated in non-LTE are $\mathrm{H}, \mathrm{He}, \mathrm{C}, \mathrm{N}, \mathrm{O}, \mathrm{S}$, and $\mathrm{Si}$. The irongroup elements, which are crucial for the radiative driving, are treated in a generalized version of the modified nebular approximation (Schmutz 1991). However, we performed test calculations in which we also explicitly computed Fe (iron) in nonLTE. These tests revealed that differences with respect to the assumption of the modified nebular approximation for Fe were negligible, and we therefore decided to keep the treatment of Fe approximate.

The line list used for the calculations consists of over $10^{5}$ of the strongest transitions of the elements $\mathrm{H}-\mathrm{Zn}$ extracted from the line list constructed by Kurucz \& Bell (1995). The wind was divided into 90 concentric shells, with many narrow shells in the subsonic region and wider shells in supersonic layers.

Other assumptions involve wind stationarity and sphericity. The latter seems to be a reasonable approximation, given that the bulk of WR stars are unpolarized (Vink \& Harries 2017). However, there are notable exceptions, involving those that are surrounded by ejecta nebulae (Vink et al. 2011b). Furthermore, polarization variability due to small-scale structure or wind clumping has been encountered in luminous blue variables (Davies et al. 2005, 2007). It has been well established that small-scale clumping of the outflowing gas has a pronounced effect on the ionization structure of both O-star and WR atmospheres (e.g. Hillier 1991). This has lead to a downward revision of empirical mass-loss rates, by a factor of $\sim 2-3$ (Moffat \& Robert 1994; Puls et al. 2008; Hamann et al. 2008; Vink \& Gräfener 2012; Ramírez-Agudelo et al. 2017).

Moreover, small-scale clumping might have a quantitative effect on the radiative driving itself as well, thereby affecting predicted mass-loss rates. The subtle issues of clumping and porosity on the mass-loss rate predictions were studied by Muijres et al. (2011), who found that whilst the impact on $\dot{M}$ can be large for certain specific clumping prescriptions, it was concluded overall that moderate clumping and porosity does not appear to change the mass-loss rate predictions substantially. In the present set of computations we therefore do not account for the effects of clumping. Nevertheless, it should be kept in mind that clumping might affect the predicted values.

\section{Parameter space}

We wish to predict mass-loss rates for He stars over a wide mass range, focusing on the $2-20 M_{\odot}$ range, but also going up to $60 M_{\odot}$ and down to $0.6 M_{\odot}$ into the regime of the subdwarf $\mathrm{O}$ (sdO) stars. The main focus of this study involves the stripped He stars of order $4 M_{\odot}$, as this is the mass range of stripped stars that fall just below that of the classical WR stars (in the range 5-20 $M_{\odot}$; Nugis \& Lamers 2000, which should represent absolute $\dot{M}$ values fairly well in the WR range). The luminosities in our study follow from the mass-luminosity relation of Gräfener et al. (2011). This leads to similar values for the lowmass stripped He stars as the models by Götberg et al. (2017).

We do not express $\dot{M}$ as a function of the Eddington parameter, as this would depend on the amount of ionized He versus $\mathrm{H}$, whilst Vink \& de Koter (2002) and Vink et al. (2011a) showed that $\dot{M}$ should hardly depend of the amount of He. However, the effective Eddington factor remains of key importance for higher mass models in the classical WR range.

The effective temperature sets the ionization stratification in the atmosphere and determines which lines are most active in driving the wind. As a result, $T_{\text {eff }}$ affects the predicted mass-loss rate. Here, we fix $T_{\text {eff }}$ to $50000 \mathrm{~K}$, which is characteristic for the best observed example of a stripped He star HD 45166 (Groh et al. 2008). We do not express the mass-loss rates of classical WR stars as a function of $T_{\text {eff }}$, as radii are highly uncertain because of envelope inflation and the possibly associated clumped nature of stellar envelopes (Gräfener et al. 2012).

Our grid was constructed to predict the mass-loss behaviour as a function of $L$ (or $M$ via the $M-L$ relation) and $Z$, which are scaled to the solar values (Anders \& Grevesse 1989).

\section{Results}

The main aim of the paper is to get a handle on the massloss rates for stripped He stars with masses just below those of classical WR stars, i.e. at about $4 M_{\odot}$. We predict a rate of $\log \dot{M}=-7.43$ and a terminal wind velocity of $2871 \mathrm{~km} \mathrm{~s}^{-1}$ for our $4 M_{\odot}$ model. This mass-loss rate is an order of magnitude smaller than that assumed by Götberg et al. (2017) through extrapolation of empirical WR stars by Nugis \& Lamers (2000).

Table 1 lists the rest of our mass-loss predictions. The initial stellar parameter columns are self-explanatory. The resulting predicted wind terminal velocities, mass-loss rates, and wind acceleration parameter $\beta$ are given in Cols. (4)-(6), respectively. The predicted mass-loss rates (Col. 5) are shown in Fig. 1. Various symbols are used to identify various $Z$ ranges. For visual clarity, not all $Z$ models feature here, but only a subset - with alternating $Z$ values - from Table 1 were plotted.

Figure 1 shows that $\dot{M}$ increases with $L$ and $Z$, as expected. Whilst the very high-mass model (with $M=60 M_{\odot}$; $\log \left(L / L_{\odot}\right)=6.4$ ) fits the relationship formed by the bulk of models in the $2-20 M_{\odot}$ range $\left(\log \left(L / L_{\odot}\right)=3.49-5.69\right.$ range $)$ very well, the very low-mass model $\left(M=0.6 M_{\odot} ; \log \left(L / L_{\odot}\right)=1.95\right)$ with $\log \dot{M}=-11.12$ falls below the general behaviour. It will therefore not be included in the mass loss versus $L$ power-law fitting.

When we compare the $M=0.6 M_{\odot}$ model to the predictions of Vink \& Cassisi (2002) for horizontal branch stars, we find $\log \dot{M}=-10.40$. However, the new dynamically consistent rate has $v_{\infty}$ a factor 2 higher, and for a constant wind momentum one would expect an $\dot{M}$ value a factor $\sim 2$ lower, i.e. $\log \dot{M} \simeq-10.70$. This is within a factor of two from the current value. Our value 
Table 1. Mass-loss predictions for the He main sequence. $T_{\text {eff }}$ is kept constant at 50000 .

\begin{tabular}{|c|c|c|c|c|c|}
\hline $\begin{array}{l}M_{\star} \\
{\left[M_{\odot}\right]}\end{array}$ & $\begin{array}{l}\log L \\
{\left[L_{\odot}\right]}\end{array}$ & $Z / Z_{\odot}$ & $\begin{array}{c}v_{\infty} \\
{\left[\mathrm{km} \mathrm{s}^{-1}\right]}\end{array}$ & $\begin{array}{c}\log \dot{M} \\
{\left[M_{\odot} \mathrm{yr}^{-1}\right]}\end{array}$ & $\beta$ \\
\hline 0.6 & 1.95 & 1 & 2326 & -11.12 & 0.70 \\
\hline \multirow[t]{3}{*}{2} & 3.49 & 1 & 2650 & -8.44 & 0.75 \\
\hline & & $1 / 3$ & 2487 & -8.90 & 0.78 \\
\hline & & $1 / 30$ & 635 & -10.18 & 0.54 \\
\hline \multirow[t]{2}{*}{3} & 3.95 & 1 & 2649 & -7.82 & 0.79 \\
\hline & & $1 / 3$ & 2655 & -8.18 & 0.81 \\
\hline \multirow[t]{3}{*}{4} & 4.26 & 1 & 2871 & -7.43 & 0.85 \\
\hline & & $1 / 3$ & 2667 & -7.72 & 0.82 \\
\hline & & $1 / 10$ & 2252 & -8.18 & 0.83 \\
\hline \multirow[t]{3}{*}{5} & 4.48 & 1 & 3039 & -7.15 & 0.88 \\
\hline & & $1 / 3$ & 2787 & -7.43 & 0.83 \\
\hline & & $1 / 10$ & 2481 & -7.80 & 0.89 \\
\hline \multirow[t]{5}{*}{8} & 4.93 & 1 & 3265 & -6.58 & 0.96 \\
\hline & & $1 / 3$ & 2714 & -6.79 & 0.87 \\
\hline & & $1 / 10$ & 2672 & -7.11 & 0.88 \\
\hline & & $1 / 33$ & 2162 & -7.50 & 0.97 \\
\hline & & $1 / 100$ & 1489 & -8.16 & 1.11 \\
\hline \multirow[t]{4}{*}{10} & 5.13 & 1 & 3472 & -6.36 & 1.00 \\
\hline & & $1 / 3$ & 2706 & -6.55 & 0.87 \\
\hline & & $1 / 10$ & 2655 & -6.85 & 0.87 \\
\hline & & $1 / 33$ & 2319 & -7.29 & 0.96 \\
\hline \multirow[t]{5}{*}{15} & 5.47 & 1 & 3790 & -5.94 & 1.04 \\
\hline & & $1 / 3$ & 2848 & -6.08 & 0.92 \\
\hline & & $1 / 10$ & 2587 & -6.33 & 0.88 \\
\hline & & $1 / 33$ & 2485 & -6.69 & 0.93 \\
\hline & & $1 / 100$ & 1985 & -7.13 & 1.08 \\
\hline \multirow[t]{6}{*}{20} & 5.69 & 1 & 3960 & -5.67 & 1.08 \\
\hline & & $1 / 3$ & 2953 & -5.80 & 0.96 \\
\hline & & $1 / 10$ & 2576 & -6.04 & 0.90 \\
\hline & & $1 / 33$ & 2504 & -6.35 & 0.92 \\
\hline & & $1 / 100$ & 2111 & -6.78 & 1.03 \\
\hline & & $1 / 333$ & 1739 & -7.418 & 1.17 \\
\hline \multirow[t]{5}{*}{60} & 6.40 & 1 & 4616 & -4.80 & 1.28 \\
\hline & & $1 / 3$ & 3507 & -4.94 & 1.10 \\
\hline & & $1 / 10$ & 2629 & -5.09 & 0.95 \\
\hline & & $1 / 33$ & 2347 & -5.31 & 0.91 \\
\hline & & $1 / 100$ & 2308 & -5.63 & 0.93 \\
\hline
\end{tabular}

for the $0.6 M_{\odot}$ model is a factor of $\sim 5$ larger than the SdO massloss predictions by Krtička et al. (2016).

We next turn our attention to the wind velocity structure parameter, $\beta$, which describes how rapidly the wind accelerates. The predicted values of $\beta$ are shown in Col. (6) of the table. $\beta$ shows a slight dependence on stellar mass, but mostly $\beta$ values are of order unity, in accordance with the O-star models of Pauldrach et al. (1986), Müller \& Vink (2008), and Muijres et al. (2012).

\section{Mass-loss recipe for He stars}

In order to determine the dependence of the mass-loss rate on $L$ and $Z$ simultaneously, we perform multiple linear regression, and we find

$\log \dot{M}=-13.3+1.36 \log \left(L / L_{\odot}\right)+0.61 \log \left(Z / Z_{\odot}\right)$

with a fitting error of $\sigma=0.11$. The formula was derived for the $Z$ range $\left(Z / Z_{\odot}\right)=1-10^{-2}$ and the mass range $2-60 M_{\odot}$, but

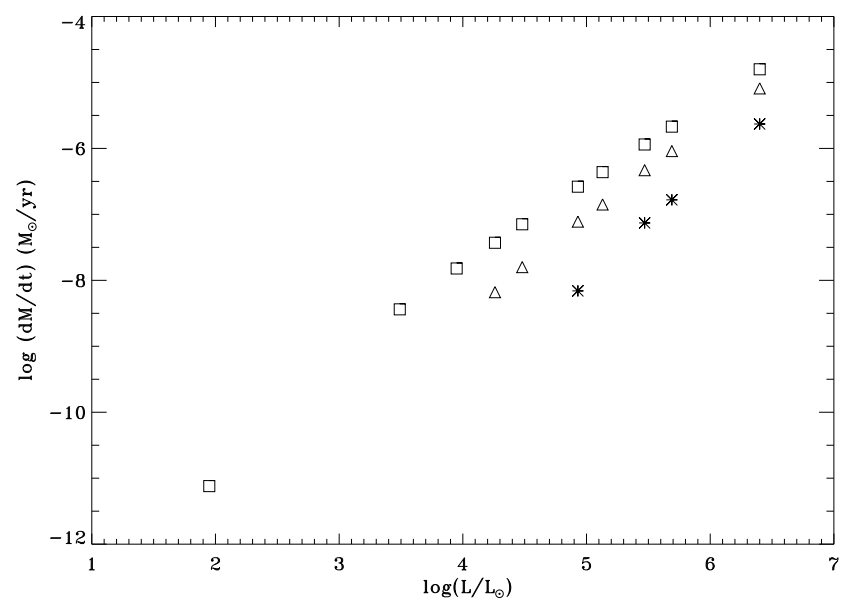

Fig. 1. Predicted mass-loss rates vs. $\log \left(L / L_{\odot}\right)$ for solar metallicity models (open squares), models of $10 \% Z_{\odot}$ (open triangles), and $1 \%$ $Z_{\odot}$ (asterisks). The high-mass models of $60 M_{\odot}$ follow the bulk of the models very well; the very low-mass models with $M=0.6 M_{\odot}$ $\left(\log \left(L / L_{\odot}\right)=1.95\right)$ fall somewhat below the general behaviour.

for reasons given earlier application of this formula is recommended only for lower-mass stripped stars, and not for classical WR stars.

The mass-loss versus mass relationship (as explored by e.g. Langer 1989) can be transformed using relevant massluminosity relationships (e.g. Gräfener et al. 2011).

\section{Discussion}

Comparing our mass-loss predictions against observed massloss rates is a non-trivial undertaking, as low-mass stripped He stars are as yet elusive.

Arguably the best example of a stripped star is HD 45166, which consists of a $4 M_{\odot}$ quasi-WR (qWR) star orbiting a B7V companion (Groh et al. 2008), which may have evolved from a $12 M_{\odot}$ primary (Götberg et al. 2017). HD 45166 has a surprisingly low (equatorial)) terminal wind velocity of $350 \mathrm{~km} \mathrm{~s}^{-1}$. For such a low $v_{\infty}$, and employing our global approach (Vink et al. 2000), we would arrive at a mass-loss rate that is an order of magnitude larger than when we use a higher value, such as for the presumed polar wind component. The agreement between the observed mass-loss rate from HD 45166 from Groh et al. (2008) and the mass-loss rates given by extrapolation of the empirical Nugis \& Lamers (2000) WR recipe is probably the main reason why Götberg et al. (2017) assumed that the Nugis \& Lamers (2000) WR mass-loss rates provide rates in the expected range for low-mass He stars, whilst we find that they are actually overestimated by an order of magnitude.

Regarding the metallicity dependence, the exponent of 0.61 is slightly less than the predicted value of 0.85 in Vink \& de Koter (2005). This is to be expected, as the earlier predictions were based on the global approach, and the difference may simply be attributed to the dependence of $v_{\infty}$ on $Z$ (Vink et al. 2001).

Our $\dot{M}-Z$ exponent is also smaller than that derived in the recent empirical study by Hainich et al. (2015), who suggest an $\dot{M}-Z$ exponent larger than unity. Given that, firstly, the Hainich et al. empirical rates were derived for a data set showing a significant scatter when expressed as a function of $L$, which suggests the Hainich et al. (2015) study misses a relevant parameter and, secondly, that our exponent was derived over a far wider range 
in $Z$ than the Hainich et al. data, we consider our lower exponent of 0.61 to be more likely close-to-correct.

Note that these $\dot{M}-Z$ exponents are predominately determined by the $\mathrm{Fe}$ abundance, and distinct from the $Z$-dependence in the empirical Nugis \& Lamers (2000) recipe. We do not consider the Nugis \& Lamers (2000) formulation physically astute, but it should still provide reasonably accurate $\dot{M}$ estimates within the actual classical WR range.

\section{Summary}

We presented mass-loss predictions from Monte Carlo radiative transfer models for stripped He stars by stellar winds or mass transfer through a companion.

- The mass-loss rate is expressed as a function of $L$ and $Z$ through multiple linear regression.

- We find $\log \dot{M}=-13.3+1.36 \log \left(L / L_{\odot}\right)+0.61 \log \left(Z / Z_{\odot}\right)$, derived for $\left(Z / Z_{\odot}\right)=1-10^{-2}$.

- Our mass-loss rates for low-mass stripped stars are an order of magnitude lower than would be found by extrapolating empirical WR recipes.

This has major implications for the search for low-mass stripped $\mathrm{He}$ stars as the progenitors of $\mathrm{SNe}$ Ibc and ionizing sources in the Universe.

\section{References}

Abbott, D. C., \& Lucy, L. B. 1985, ApJ, 288, 679

Abbott, B. P., Abbott, R., Abbott, T. D., et al. 2016, Phys. Rev. Lett., 116, 061102 Anders, E., \& Grevesse, N. 1989, Geochim. Cosmochim. Acta, 53, 197 Belczynski, K., Bulik, T., Fryer, C. L., et al. 2010, ApJ, 714, 1217 Cassata, P., Le Fèvre, O., Charlot, S., et al. 2013, A\&A, 556, A68 Castor, J., Abbott, D. C., \& Klein, R. I. 1975, ApJ, 195, 157 Davies, B., Oudmaijer, R. D., \& Vink, J. S. 2005, A\&A, 439, 1107 Davies, B., Vink, J. S., \& Oudmaijer, R. D. 2007, A\&A, 469, 1045 de Koter, A., Schmutz, W., \& Lamers, H. J. G. L. M. 1993, A\&A, 277, 561 Eldridge, J. J., \& Vink, J. S. 2006, A\&A, 452, 295
Georgy, C., Ekström, S., Eggenberger, P., et al. 2013, A\&A, 558, A103 Götberg, Y., de Mink, S. E., \& Groh, J. H. 2017, A\&A, in press DOI: $10.1051 / 0004-6361 / 201730472$

Gräfener, G., \& Vink, J. S. 2015, A\&A, 578, L2

Gräfener, G., Vink, J. S., de Koter, A., \& Langer, N. 2011, A\&A, 535, A56

Gräfener, G., Owocki, S. P., \& Vink, J. S. 2012, A\&A, 538, A40

Groh, J. H., Oliveira, A. S., \& Steiner, J. E. 2008, A\&A, 485, 245

Hainich, R., Pasemann, D., Todt, H., et al. 2015, A\&A, 581, A21

Hamann, W.-R., Feldmeier, A., \& Oskinova, L. M. 2008, Clumping in Hot-Star Winds, Proc. of an Internaional Workshop held in Potsdom, Germany

Hillier, D. J. 1991, A\&A, 247, 455

Krtička, J., Kubát, J., \& Krtičková, I. 2016, A\&A, 593, A101

Kurucz, R. L. \& Bell, B. 1995, Atomic Line Data Kurucz CD-ROM No. 23 (Cambridge, Mass.: Smithsonian Astrophysical Observatory)

Langer, N. 1989, A\&A, 220, 135

Langer, N. 2012, ARA\&A, 50, 107

Limongi, M., \& Chieffi, A. 2006, ApJ, 647, 483

Lucy, L. B., \& Solomon, P. M. 1970, ApJ, 159, 879

Moffat, A. F. J., \& Robert, C. 1994, ApJ, 421, 310

Muijres, L. E., de Koter, A., Vink, J. S., et al. 2011, A\&A, 526, A32

Muijres, L. E., Vink, J. S., de Koter, A., Müller, P. E., \& Langer, N. 2012, A\&A, 537, A37

Müller, P. E., \& Vink, J. S. 2008, A\&A, 492, 493

Müller, P. E., \& Vink, J. S. 2014, A\&A, 564, A57

Nugis, T., \& Lamers, H. J. G. L. M. 2000, A\&A, 360, 227

Pauldrach, A. W. A., Puls, J., \& Kudritzki, R. P. 1986, A\&A, 164, 86

Podsiadlowski, P., Joss, P. C., \& Hsu, J. J. L. 1992, ApJ, 391, 246

Puls, J., Vink, J. S., \& Najarro, F. 2008, A\&ARv, 16, 209

Ramírez-Agudelo, O. H., Sana, H., de Koter, A., et al. 2017, A\&A, 600, A81

Sobral, D., Matthee, J., Darvish, B., et al. 2015, ApJ, 808, 139

Szécsi, D., Langer, N., Yoon, S.-C., et al. 2015, A\&A, 581, A15

Schmutz, W. 1991 in Stellar Atmospheres: Beyond Classical Models, eds. L. Crivellari, I. Hubeny, \& D. G. Hummer, NATO ASI Series C, 341, 191

Vink, J. S. 2015, in Very Massive Stars in the Local Universe, Astrophys. Space Sci. Lib., 412, 77

Vink, J. S., \& Cassisi, S. 2002, A\&A, 392, 553

Vink, J. S., \& de Koter, A. 2002, A\&A, 393, 543

Vink, J. S., \& de Koter, A. 2005, A\&A, 442, 587

Vink, J. S., \& Gräfener, G. 2012, ApJ, 751, L34

Vink, J. S., \& Harries, T. J. 2017, A\&A, 603, A120

Vink, J. S., de Koter, A., \& Lamers, H. J. G. L. M. 1999, A\&A, 345, 109

Vink, J. S., de Koter, A., \& Lamers, H. J. G. L. M. 2000, A\&A, 362, 295

Vink, J. S., de Koter, A., \& Lamers, H. J. G. L. M. 2001, A\&A, 369, 574

Vink, J. S., Muijres, L. E., Anthonisse, B., et al. 2011a, A\&A, 531, A132

Vink, J. S., Gräfener, G., \& Harries, T. J. 2011b, A\&A, 536, A10

Yoon, S.-C., Gräfener, G., Vink, J. S., Kozyreva, A., \& Izzard, R. G. 2012, A\&A, 544, L11 